\title{
Advanced Logic Gates for Ultrafast Network Interchanges
}

\author{
Mohammed N. Islam \\ Department of Electrical Engineering and Computer Science \\ University of Michigan, Ann Arbor, Michigan 48109-2122
}

\begin{abstract}
By overcoming speed bottlenecks from electronic switching as well as optical/electronic conversions, all-optical logic gates can permit further exploitation of the nearly $40 \mathrm{THz}$ of bandwidth available from optical fibers. We focus on the use of optical solitons and alloptical logic gates to implement ultrafast "interchanges" or switching nodes on packet networks with speeds of $100 \mathrm{Gbit} / \mathrm{s}$ or greater. For example, all-optical logic gates have been demonstrated with speeds up to $200 \mathrm{Gbit} / \mathrm{s}$, and they may be used to decide whether to add or drop a data packet. The overall goal of our effort is to demonstrate the key enabling technologies and their combination for header processing in $100 \mathrm{Gbit} / \mathrm{s}$, time-division-multiplexed, packed switched networks.

Soliton-based fiber logic gates are studied with the goal of combining attractive features of soliton-dragging logic gates, nonlinear loop mirrors and erbium-doped fiber amplifiers to design logic gates with optimum switching energy, contrast ratio and timing sensitivity. First, the experimental and numerical work studies low-latency soliton logic gates based on frequency shifts associated with cross-phase modulation. In preliminary experiments, switching in $15 \mathrm{~m}$ long low-birefringent fibers has been demonstrated with a contrast ratio of 2.73:1. Using dispersion-shifted fiber in the gate should lower the switching energy and improve the contrast ratio. Next, the low-birefringent fiber can be cross-spliced and wrapped into a nonlinear optical loop mirror to take advantage of mechanisms from both soliton dragging and loop mirrors. The resulting device can have low switching energy and a timing window that results from a combination of soliton dragging and the loop mirror mechanisms.
\end{abstract}

\section{INTRODUCTION}

Implementing an ultrafast information superhighway requires wide roadways and high-speed interchanges. Optical fibers, with their $40 \mathrm{THz}$ of potential bandwidth, provide the wide roadways. However, we can only tap a tiny fraction (a few gigabits-per-second) of this bandwidth because we use electronics at the 
interchanges (switching nodes) where information enter and exit the network. We propose to overcome the electronic switching bottlenecks by using light-controllinglight (all-optical) switching to complement optical transport of information.

Our research falls in the general category of ultrafast, time-division-multiplexed (TDM), serial optical processing in networks. Serial optical processing is particularly attractive when information enters and exits in optical format on fibers. Also, TDM is advantageous because it permits a trully all-digital system with dynamic allocation of bandwidth; i.e., by time-interleaving various users, idle channels in the network can be avoided. High-speed TDM is important for alloptical networks for several reasons. First, TDM is more natural for self-routing packet switching, and it permits us to take advantage of the over 25 years of experience that we have from the electronics arena. Second, this work complements other wavelength-division-multiplexed (WDM) research, since highspeed TDM can eventually be used to upgrade the speeds of individual WDM channels. Finally, high-speed TDM may simplify the system architecture and protocol by avoiding the challenges of parallel programming required for a multichannel WDM systems.

All-optical switching means that one light beam controls the passage or modulates another light beam by interacting in a nonlinear medium. Light-light manipulations can be very fast if nonresonant, virtual optical interactions are used: i.e., the interaction is through deformation of electron clouds or wave functions rather than through the generation of electron-hole pairs, which must then recombine before the switch is reused. There are several compelling reasons for turning toward alloptical switching for serial, terabit-rate processing. First, speeds exceeding 50 $\mathrm{Gbit} / \mathrm{s}$ can be achieved, which is beyond where electronic systems might be expected to operate. Second, by processing optically we can remove bottlenecks from converting between optics and electronics, which is particularly important if the information enters and exits in optical format. Third, electronic gates or devices that absorb light tend to generate heat, which becomes a major problem at very high bit rates. On the other hand, if nonresonant interactions are exploited, then at least in principle heat dissipation is not a limiting factor. Finally, and perhaps most importantly, all-optical gates enable us to distribute intelligence throughout the network and implement "optical control" in addition to optical transport. For example, since the data and routing information can be in the same optical format, the destination can be encoded with the data. This enables what is commonly called "self-routing packet switching," which means a packet can route itself through the network. To implement such a network electronically requires that the optical information be converted to electrical signals, which runs into the optical/electronic conversion bottlenecks.

Considerable research is focused on "solitons" in optical fibers and their applications to long-distance telecommunications and ultra-high-speed information processing and networks. Solitons are robust pulses that propagate nearly distortion-free for long distance in fibers [1]. For example, Mollenauer [2] has transmitted solitons virtually error-free over 13,000 kilometers at $20 \mathrm{Gbit} / \mathrm{sec}$. In addition, Islam [1] has demonstrated the world's fastest logic gates based on solitons in special types of fibers. With the considerable experimental and theoretical studies on soliton transmission and switching in the United States as well as Europe and Japan, there is a growing need for integration of soliton generation, propagation and switching for network applications.

Solitons arise in optical fibers in the anomalous group velocity dispersion regime (wavelengths longer than $1.3 \mu \mathrm{m}$ ), and solitons represent a balance between the 
nonlinearity and the group-velocity dispersion in the fiber. Because of these two counteracting effects, the soliton is a stable pulse with a restoring force that makes it behave in many ways like a single particle or fundamental data unit. There are several advantages of usings solitons for switching. First, we can avoid the deleterious effects of group-velocity dispersion and nonlinearity, which become increasingly important for short pulses, since the two effects are kept in balance. Second, the particle-like nature of solitons permits cascadable switching (where output pulse looks like input pulse) with high-contrast ratio (since the entire pulse switches). Third, we can exploit unique properties of solitons for switching. For example, our group has demonstrated switches based on modulational instability, soliton-trapping and soliton-dragging, all of which only occur in the soliton regime. Finally, soliton switching aligns the effort with long-haul transmission developments, permitting us to take advantage of new technologies that are developed for transmission in switching.

We aim to take advantage of all-optical switching and solitons to implement key enabling components for ultrafast TDM networks. We try to combine attractive features of soliton-dragging logic gates, nonlinear loop mirrors and erbium-doped fiber amplifiers to design logic gates with optimum switching energy, contrast ratio and timing sensitivity. First, the experimental and numerical work studies lowlatency soliton logic gates based on frequency shifts associated with cross-phase modulation. In preliminary experiments, switching in $15 \mathrm{~m}$ long low-birefringent fibers has been demonstrated with a contrast ratio of $2.73: 1$. Using dispersionshifted fiber in the gate should lower the switching energy and improve the contrast ratio. Next, the low-birefringent fiber can be cross-spliced and wrapped into a nonlinear optical loop mirror to take advantage of mechanisms from both soliton dragging and loop mirrors. The resulting device can have low switching energy and a timing window that results from a combination of soliton dragging and the loop mirror mechanisms.

\section{VARIOUS DESIGNS OF ALL-OPTICAL LOGIC GATES}

To be able to make decisions in all-optical networks at add/drop multiplexer points, we need all-optical logic gates to enable header processing. For digital applications, the logic gates must be cascadable (i.e., the ability of one gate to drive a similar gate), have fan-out and be Boolean complete (i.e., the ability to create gates that perform the complete range of Boolean operations). In addition, it is advantageous if the devices have phase-insensitive operation, logic-level restoration and input/output isolation. Several categories of devices have emerged as satisfying some or all of the above requirements. For example, nonlinear optical loop mirrors (NOLM's) have been used in demultiplexers and have been demonstrated to have wide timing windows for particular designs. Also, soliton-dragging logic gates (SDLG's), which are based on the nonlinear interaction between orthogonally polarized pulses, have been demonstrated in various logic operations. Furthermore, with the recent advances in erbium-doped fiber amplifiers (EDFA's), there is increased interest in using EDFA's with logic gates to lower the switching energy, increase the fan-out, and perhaps even contribute to the switching function in the gates. We are studying designs of logic gates that in some fashion combine the best traits of NOLM's, SDLG's and EDFA's to render low enough switching energy that the devices can be driven by our erbium-doped fiber lasers (EDFL's). 
We start by reviewing NOLM's and SDLG's, and then we show some hybrid schemes using novel low-birefringent (low-bi) fiber recently demonstrated in our laboratory.

\section{Nonlinear Optical Loop Mirrors}

One example of a routing switch in fibers is a nonlinear Mach-Zehnder interferometer, where switching occurs when the phase of one arm is shifted by $\pi$ relative to the phase of the other arm. Long lengths of fibers are required to reduce the switching energy because of the weak fiber nonlinearity. However, since the interferometer must be stable to within a fraction of a wavelength, it is difficult to use two physically separate fibers to implement a long interferometer. An implementation of the interferometer that has received much attention is based on a nonlinear Sagnac interferometer or a nonlinear optical loop mirror (NOLM)[3]. As depicted in Fig. 1, the NOLM consists of a four-port directional coupler in which two ports on one side are connected by a loop of fiber. The two arms of the interferometer correspond to the two counter-propagating directions around the loop, and this configuration is very stable since both arms involve exactly the same optical path.

When the coupler divides the input equally, the NOLM acts as a perfect mirror. By adding a signal beam that is orthogonal either in frequency or polarization, the NOLM can also act as a three-terminal switch. In particular, the signal beam propagates only in one direction around the NOLM, and it phase shifts the control pulse in that direction through cross-phase modulation due to the nonlinear index of refraction. Therefore, when the control and signal pulses are incident, an output results from the NOLM. This output is maximum for a $\pi$-phase shift through cross-phase modulation, and the output varies as a squared sinusoidal function of the phase shift [4].

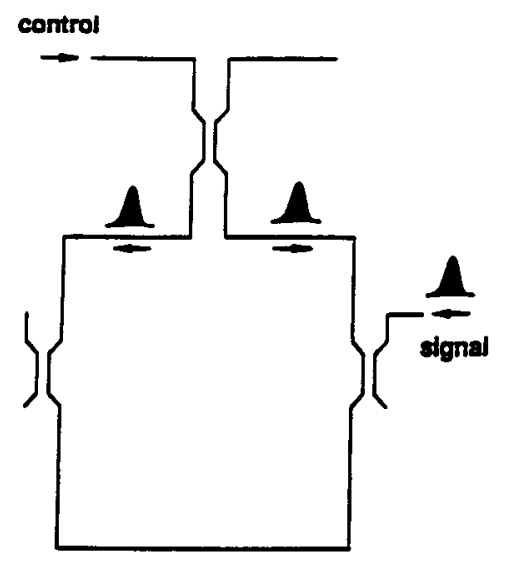

FIGURE 1. Schematic of a nonlinear optical loop mirror in which orthogonally polarized control and signal pulses interact and the signal pulses enter and exit through separate polarizing beam splitters. 


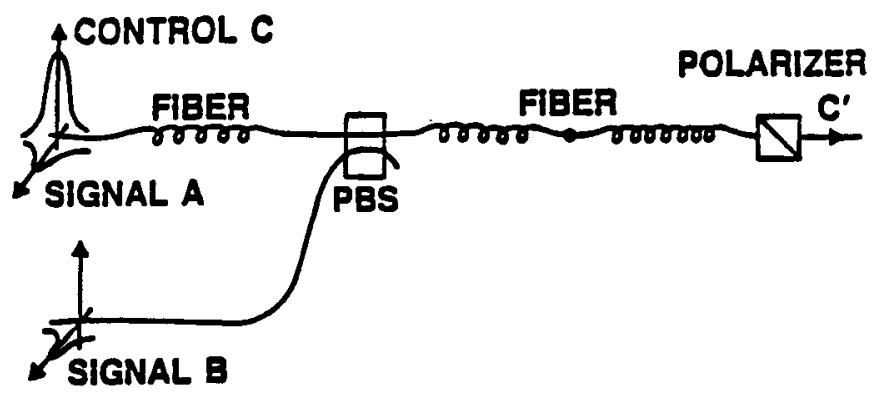

FIGURE 2. Schematic of a soliton-dragging NOR gate with control or power supply $C$ along one axis and signals $A$ and $B$ polarized orthogonally (PBS=polarizing beam splitter).

Various configurations and applications of NOLM's have been demonstrated over the past several years. As a single-input device (no signal), the NOLM acts as a fast saturable absorber or a pulse shaper. For example, the nonlinear transmission of the NOLM can be used for noise or background removal since the low-intensity signals are reflected by the NOLM while the high-intensity signals are propagated. Moreover, the NOLM can act as a modelocker in a laser because the nonlinear device prefers short pulses with high intensity over continuous waves with low intensity. When a signal is added, the three-terminal switch can be used for demultiplexing or logic operations. Demultiplexing plucks out selected signals in an incoming bit stream, while logic operations enable computations or decisionmaking capabilities.

\section{Soliton Logic Gates}

Soliton-dragging logic gates (SDLGs) have been shown to satisfy all requirements for devices in a digital optical processor [5]. In soliton dragging, the speed or propagation time of the control pulse depends on whether it propagates alone or if it is accompanied by one or both of the signals. Physically, when the two pulses are coincident, they interact through cross-phase modulation and "chirp" each other or change each others center frequency. This frequency change leads to a change in velocity because of the group-velocity dispersion in the fiber.

A schematic of a soliton-dragging NOR-gate is shown in Fig. 2. The gate consists of two lengths of fibers coupled through a beam splitter and ending in a polarizer. The control or power supply pulses propagate along one axis, and the signal pulses are launched into the orthogonal axis. We assume that the system is clocked and digital, and the logic is based on time-shift keying (i.e. , the control pulse arriving within the clock window corresponds to a Boolean one, and any other result is a Boolean zero). Soliton-dragging NOR gates have been demonstrated with switching energies approaching a picojoule and speeds as high as $200 \mathrm{Gbit} / \mathrm{s}$.

At the boundary of the all-optical system, another type of logic gate known as a soliton-trapping AND-gate is used [1]. Soliton-trapping gates do not exhibit gain or cascadability, but they are sensitive to the timing of the input pulses and provide 
an energy contrast at the output. Consequently, the trapping gates convert the time-shift keyed signal to an amplitude-shift keyed output, which can then be detected and converted to electronic format.

For time-division multiplexed systems with single channel speeds of $100 \mathrm{Gbit} / \mathrm{sec}$ and beyond, picosecond or sub-picosecond pulses will be required. For these short pulses it has been shown theoretically that it is energetically advantageous to use SDLG's rather than NOLM's or NLDC's for switching [6]. Whereas NOLM's and NLDC's require at least a $\pi$-phase shift from the interaction between two pulses to switch state, experiments using 0.5 ps pulses in SDLG's require only about a $\pi / 20$ phase shift. In fact, for fiber device lengths less than about a kilometer, SDLG's require less switching energy for pulse widths under about $5 \mathrm{ps}$. Therefore, for the 20 to $35 \mathrm{ps}$ pulses that are used for $2.5 \mathrm{Gbit} / \mathrm{sec}$ switching, it is favorable to use Mach-Zehnder interferometers. However, for the demanding task of $100 \mathrm{Gbit} / \mathrm{sec}$ time-domain switching using about lps pulses, SDLG's have an energy advantage. Of course, the optimum solution may lie somewhere in between the two designs, as described below.

\section{Low-Latency Soliton Logic Gate using Low-Bi Fiber}

As an example of combining EDFA's with soliton logic gates, we have begun to explore how the latency of the fiber devices can be reduced by using EDFA's. In preliminary experiments, we have demonstrated switching in $15 \mathrm{~m}$ of fiber, which is more than an order of magnitude shorter than existing gates. A schematic of the low-latency switch is shown in Fig. 3. We launch two orthogonally polarized pulses (control and signal) into the device, and use a polarizer to observe only the control at the output. With the signal blocked, the control propagates through the fiber and emerges at the output. However, when the signal is added, the two pulse interact through cross-phase modulation and walk-off due to the birefringence, resulting in a frequency shift of the control. The frequency shifted control is then blocked by the frequency filter at the output. Hence, the device acts as an inverter for the signal pulse. The EDFA is used to increase the intensity of the pulses, and a low-birefringent fiber is chosen to maximize the interaction length or walk-off length between the orthogonally-polarized pulses. This low-bi fiber is polarization maintaining, which is critical for avoiding phase-sensitive switching. The frequency filter leads to an energy contrast at the output after the pulses interact.

To explore the parameter range for such a switch, we solve the coupled nonlinear Schroedinger equation using a split-step Fourier transform technique. The simulations include group-velocity dispersion, self-phase modulation, cross-phase

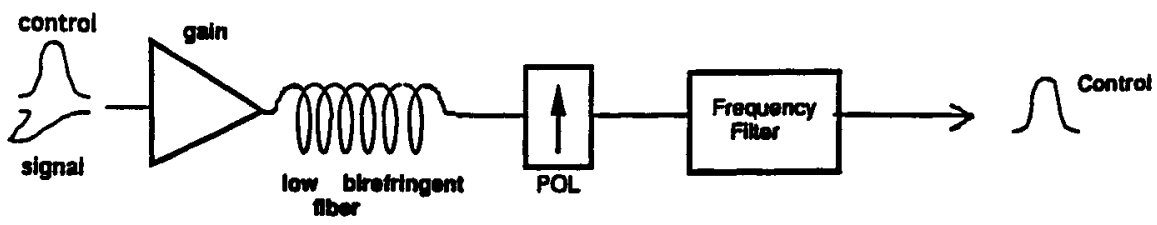

FIGURE 3. Configuration for a soliton logic gate with a discrete amplifier used to increase the amplitude at the input ( $P O L=$ polarizer). 


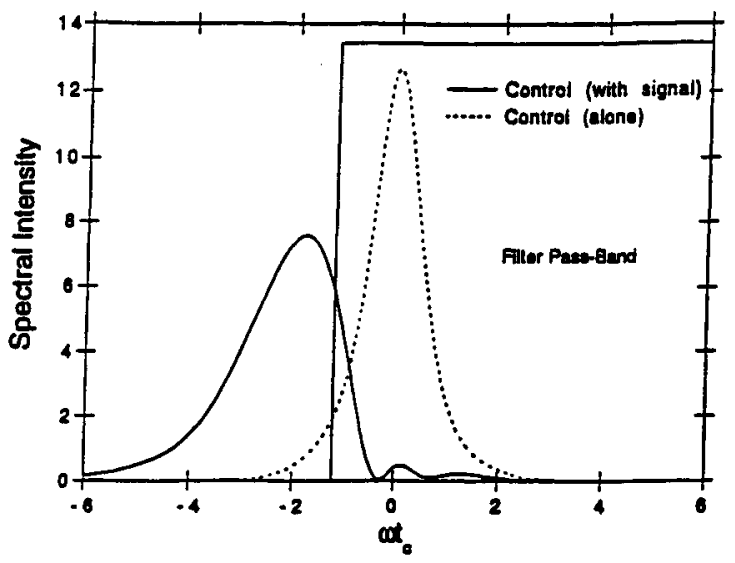

FIGURE 4. Spectrum of control pulse with and without the signal pulse for the configuration of Figure 3 . Much of the control spectrum is shifted outside of the filter pass band for a contrast ratio of 6.5:1.

modulation, birefringence and walk-off, and soliton self-frequency shift, although we find that the self-frequency shift is not crucial for the gate operation. After the fiber we add a frequency filter that has a sharp cut-off near the long wavelength edge of the input pulse spectrum. The filter can either be a high frequency pass or bandpass filter with a bandwidth wider than the input spectral width. Such a frequency filter could be implemented with multi-layered dielectric coatings or a prism filter with a slit or knife-edge between the two prisms. Also, we launch the two orthogonally-polarized pulses separated at the input so they overlap completely about mid-way down the fiber.

As an example, Fig. 4 shows the spectrum after the fiber for the control pulse with and without the signal pulse. Whereas the spectrum of the control pulse alone is similar to the input spectrum, adding the signal pulse shifts most of the control pulse spectrum. The filter pass band is shown by the dashed curve, and after integrating the output following the filter we find an energy contrast of 6.3:1. Figure 5 shows the signal input and control output pulse (without the signal present) intensities in time, verifying cascadable operation and fan-out. The control pulse output is broadened by approximately $2 \%$ because of propagation in the beginning of the fiber and the output filter, which trims off the long-wavelength tail of the pulse. Also, passage through the frequency filter leads to a slight asymmetrizing of the control pulse in time. Note that the control and signal pulses are severly distorted in time when they interact in the fiber, but that corresponds to the off-state for the switch. Finally, Fig. 6 shows the energy contrast ratio versus separation between the control and signal pulses at the input normalized to the pulse width. This gives a measure of the timing sensitivity of the logic gate, and we find a full-width half maximum value for the contrast ratio timing curve of approximately a pulse width.

To experimentally test the low-latency logic gate, we use the configuration in Fig. 7. The laser source is a passively modelocked color center laser, which we expect eventually to replace with an EDFL. The output of the laser is amplified in an $\mathrm{Er} / \mathrm{Yb}$ co-doped amplifier, which is pumped by a Nd:YAG laser, and we are careful to minimize the pulse distortion. The control and signal pulses are then split and recombined, and the timing can be adjusted between the two by a delay stage. The 


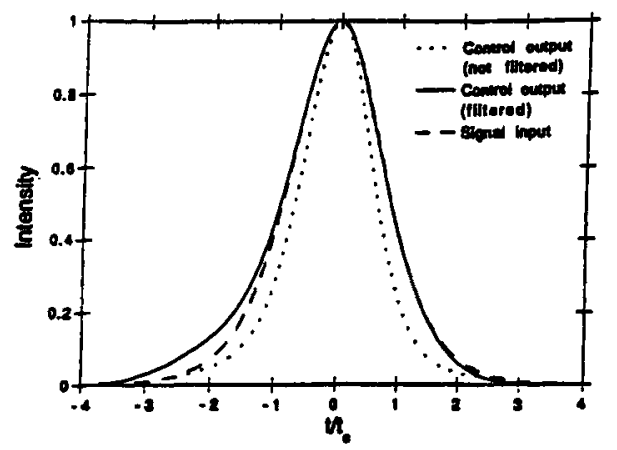

FIGURE 5. Normalized intensity of the control pulse before (dotted curve) and after (solid curve) the frequency filter with no signal pulse present. The filtered pulse broadens only by $2 \%$ from the input in this case. Because the output is similar to the signal input (dashed curve), the switch is cascadable.

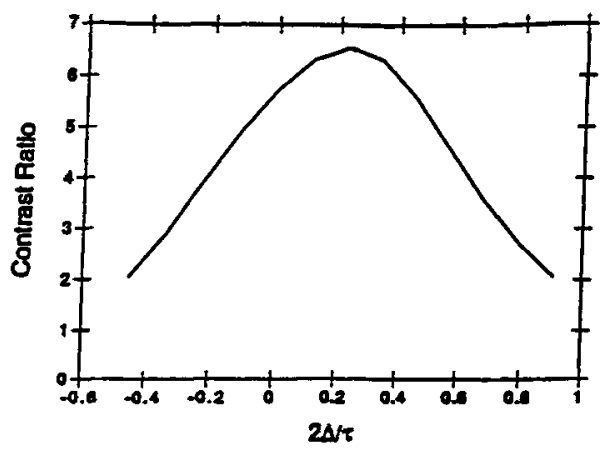

FIGURE 6. Contrast ratio versus input pulse separation. The timing window for the contrast ratio to reach half its maximum value in this case is one pulse width.

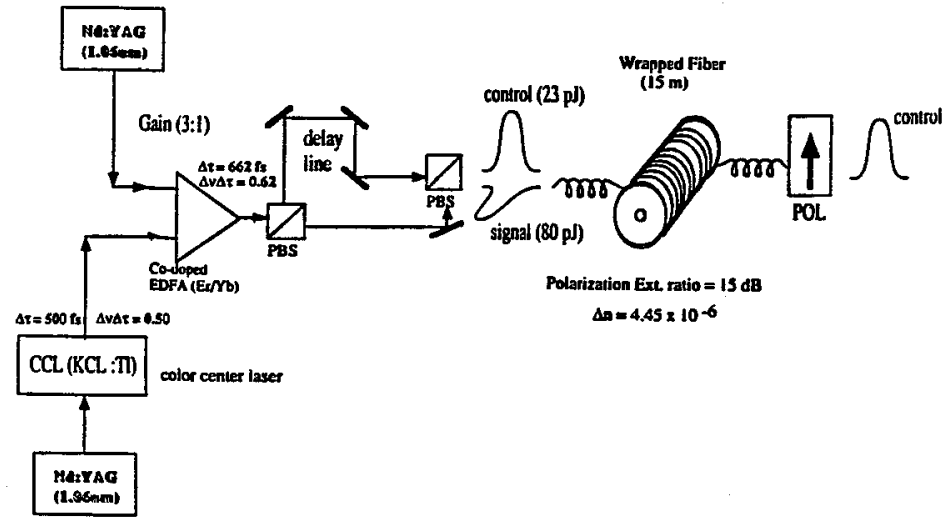

FIGURE 7. Experimental configuration for Low-Bi, low-latency soliton logic gate ( $\mathrm{CCL}=$ color center laser, $\mathrm{PBS}=$ polarization beam splitter, $\mathrm{POL}=$ polarizer) 


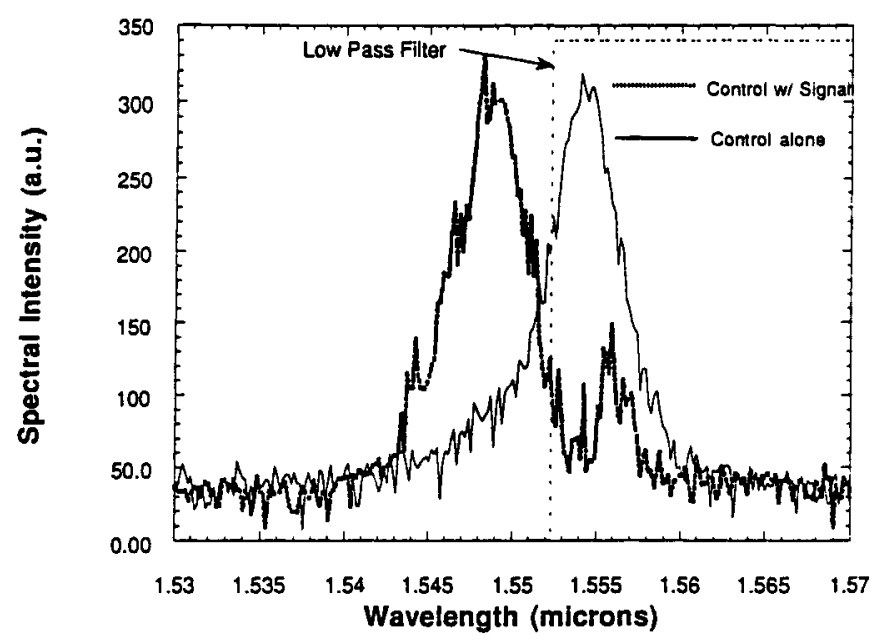

FIGURE 8. Preliminary experimental data showing shift of control spectrum with the addition of the signal pulse. The control energy is 23pJ while the signal energy is 80pJ, and the frequency shift at the peak is $0.87 \mathrm{THz}$. With the frequency fitter shown by the dotted curve, the contrast ratio is 2.73:1.

output after the polarizer is sent to a Fabry-Perot interferometer and a grating spectrometer.

A key component used in our experiments is a novel low-bi, polarization maintaining fiber, which is carefully wrapped on aluminum mandrel. We start with a low-birefringent (background birefringence less than $10^{-7}$ ) fiber, and then carefully wrap it on the mandrel. We avoid scrambling of the axes by being careful not to twist the fiber during wrapping, and the resulting birefringence is set by the diameter of the mandrel (i.e., the bend-induced birefringence). In our experiments, we achieve a birefringence of $\sim 2.3 \times 10^{-6}$ while maintaining a polarization extinction ratio of $15 \mathrm{~dB}$. Thus, even with this low birefringence, we still effectively have a polarization-maintaining fiber. The low-birefringence is important for increasing the interaction length, while the polarization maintaining property is important for avoiding phase-sensitive switching.

Preliminary data from this experiment is included in Fig. 8. The control spectra with and without the signal clearly demonstrate the frequency shift, with the peak shift being about $0.87 \mathrm{THz}$. In this experiment the signal energy is $80 \mathrm{pJ}$ and the control energy is $23 \mathrm{pJ}$, while the pulse width is $662 \mathrm{fsec}$. If we place a frequency filter as shown by the dotted curve, then we would expect a contrast ratio of 2.73:1. Simulations of these conditions show that the peak frequency shift should be $0.62 \mathrm{THz}$ with a contrast ratio on the order of $6.62: 1$. Hence, we do see the expected frequency shift, but our contrast is degraded (perhaps because of wings on the spectrum due to higher gain in EDFA's at shorter wavelengths).

We want to improve on the preliminary results by focusing on increasing the contrast ratio and decreasing the switching energy. For example, the current design uses standard fiber with a zero dispersion wavelength of $1.3 \mu \mathrm{m}$. On the other hand, if we use a dispersion shifted fiber with a zero dispersion around $1.52 \mu \mathrm{m}$, then we expect much improved behavior. Our optimum simlations show that we should be able to reduce the switching energy closer to $16 \mathrm{pJ}$ and obtain a contrast 
ratio as good as 8.86:1. On the other hand, the fiber length increases from $15 \mathrm{~m}$ up to $28 \mathrm{~m}$ with the dispersion shifted fiber, which is still an order of magnitude shorter than previous demonstrations.

\section{New Twist on NOLM: Low-Bi-NOLM}

As mentioned earlier, one of our goals is to combine NOLM's, SDLG's and EDFA's to find a design that uses the best features of all these parts. The previous section discussed using polarization-maintaining, low-bi fiber in a single-pass, open-loop, switch. If we combine two such fibers in an interferometric loop configuration, we obtain a new design of a low-birefringent fiber NOLM (Low-BiNOLM) that begins to blend aspects of SDLG's and NOLM's. As detailed below, the advantage of the Low-Bi-NOLM is lower switching energy with reduced sensitivity to timing fluctuations.

A schematic of the Low-Bi-NOLM is illustrated in Fig. 9. This is similar to the design of NOLM's by Moores, et al. [7], and Whitaker, et al. [4], but it incorporates the long interaction length between orthogonally polarized solitons of the low-bi fiber that we have recently developed. The low-bi fiber dramatically reduces the number of axos-exchanging splices required and the switching energy of the device. The control pulse propagates through this Sagnac interferometer, and when propagating by itself it is fully reflected at the output. We adjust the control energy in each fiber to be an $\mathrm{N}=1$ fundamental soliton to avoid any pulse shape distortions. The signal pulse is inserted and extracted using polarizing beam splitters, and the pulse only propagates in the clockwise direction. When the signal pulse is added and interacts with the clockwise traveling control pulse through cross-phase-modulation, the interferometer is imbalanced and an output results. We use two lengths of polarization maintaining low-bi fiber and flip the slow and fast axes when splicing them together so the pulses pass through each other twice [7]. To increase the fan-out of this gate, we could also use EDFA's at the input or output of the device.

To study the performance of the Low-Bi-NOLM, we performed preliminary numerical simulations solving the coupled nonlinear Schroedinger equation. For example, we use a $287 \mathrm{~m}$ total length of dispersion shifted fiber $(D=1 \mathrm{ps} /(\mathrm{nm}-\mathrm{km}))$ with a birefringence of $3 \times 10^{-6}$. For $0.5 p s e c$ pulses, we use a $5.3 \mathrm{pJ}$ signal pulse and $4.7 \mathrm{pJ}$ control pulse. The soliton period is $100 \mathrm{~m}$, and the walk-off length is about half a soliton period; since the total length is almost three soliton periods, we must use solitons to avoid deleterious effects from group-velocity dispersion. Adding the signal pulse leads to a net transmission of $78 \%$ of the control pulse with almost no broadening (the wings of the pulse differ slightly). For instance, Fig. 10 shows the temporal intensity of the input and switched output, and we see that the switch is cascadable. Instantaneous switching without pulse break-up occurs primarily due to the uniform phase shift across the pulse, which is partly due to the use of solitons.

In addition to the lower energy requirements, a main feature of the Low-BiNOLM is the finite timing window over which switching occurs. For the above design, Fig. 11 shows the timing sensitivity of the Low-Bi-NOLM by plotting the transmission versus initial separation. The full-width-half-maximum of the timing curve is about 3.3 pulse widths, which is larger than the roughly one pulse width range of the previously described soliton logic gate. In addition, it should be clear that further improvements can be made to increase the timing window and to lower 
M. N. Islam 615

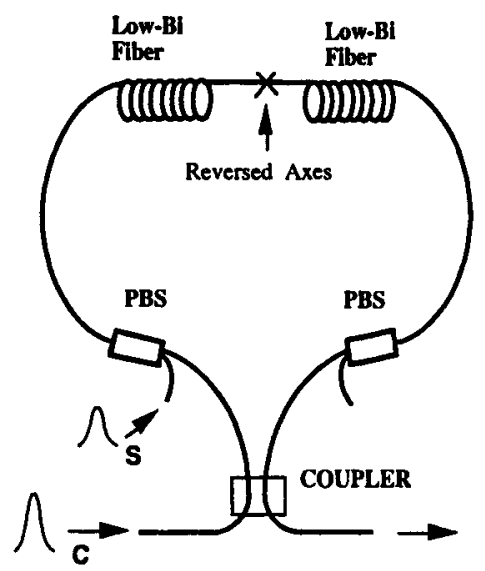

FIGURE 9. Schematic of the Low-Bi-NOLM using two segments of low-bi fiber that are spliced with their axes crossed (PBS=polarizing beam splitter, Low-Bi=low birefringent, polarization maintaining fiber).

Intensity of Pulse-in and Pulse-out vs. t/tc

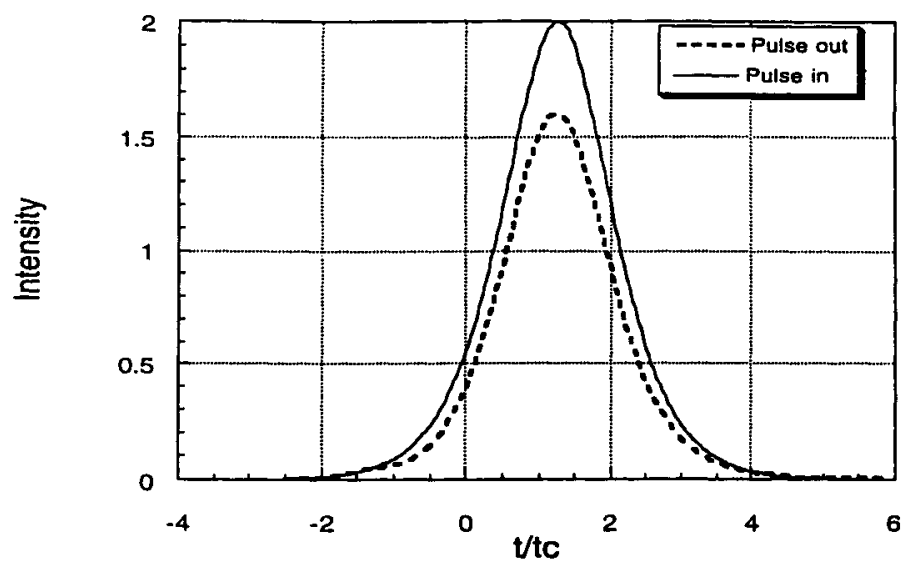

FIGURE 10. Temporal intensity of input and output pulses from Low-Bi-NOLM. The cascadability of the switch is confirmed because the pulse widths are roughly equal, although there is a slight broadening of the wings. 


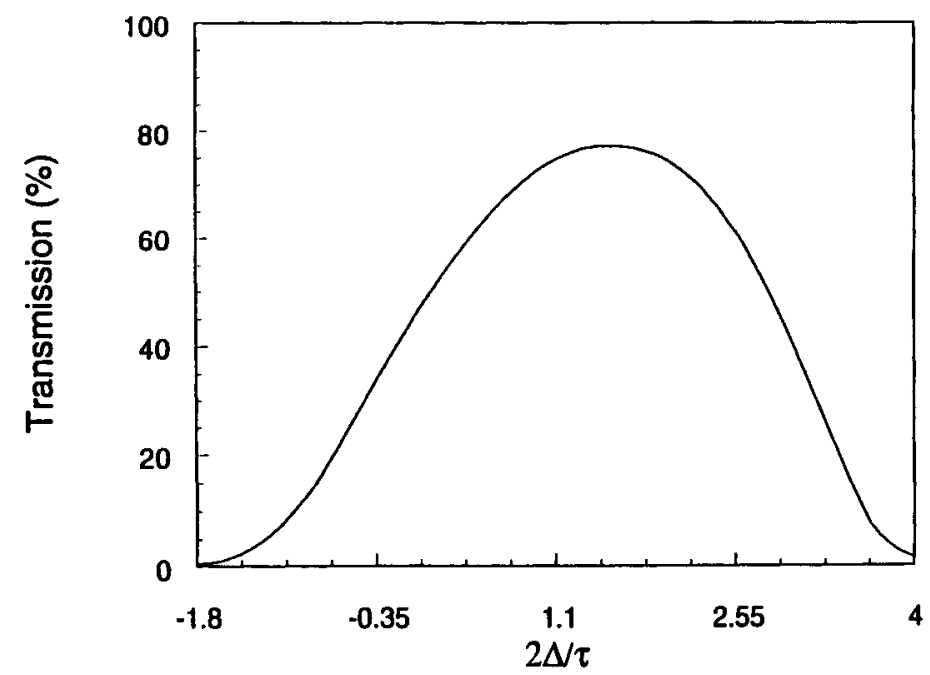

FIGURE 11. Test of the timing sensitivity of the Low-Bi-NOLM by plotting the transmission coefficient versus initial separation between pulses. The full-width-halfmaximum is about 3.3 pulse widths.

the switching energy. Namely, the timing window is controlled by the length of each of the segments of fiber, while the switching energy can be lowered by including more sections of cross-spliced fibers, thereby accumulating the required $\pi$-phase shift over more segments.

Even from our preliminary design efforts we have found a set of design rules that govern the optimization of the Low-Bi-NOLM. The most obvious rule is that we need a total of $\pi$-phase shift to convert from destructive to constructive interference at the coupler. Second, we need a nearly uniform phase shift across the pulse to obtain complete switching without pulse break-up. Third, we need a minimum level of birefringence to avoid soliton trapping and the associated pulse shape distortions as the pulses pull on each other. In particular, we find that we need $\mathrm{d}>0.6$, where $\mathrm{d}$ is the normalized birefringence in standard soliton units. Fourth, we find that to minimize pulse shape distortions we want the walk-off length to be shorter than half of the soliton period. Finally, we must keep the control and signal amplitudes $\mathrm{A}<1.5$ to avoid higher order solitons. These combined requirements lead to a multi-segment design with low switching energy and cascadable operation.

One significant issue is the manner in which the current design uses both soliton dragging and mechanisms in the NOLM. This is best illustrated by the timing curve of Fig. 11. In the case of the NOLM walk-through alone, the timing curve is generally symmetric (c.f., Whitaker, et al. [4]) and roughly equal to the number of walk-throughs: for this example, it would be roughly two pulse widths wide. However, we see in Fig. 11 that the timing curve is broader and slightly asymmetric toward the side corresponding to perfect overlap at the input. In fact, this asymmetry and broadening is occuring because of soliton dragging when the pulses overlap within plus/minus a half a pulse width at the input of either segment of fiber. We believe that this effect can be accentuated when there are an odd number of cross-spliced segments, or we could highten the effect by placing an EDFA at the mid-point in the single splice case. 


\section{SUMMARY}

Our research provides key enabling building blocks for $100 \mathrm{Gbit} / \mathrm{s}$ rate transmission systems, switched local- and wide-area networks, and serial optical processing. In particular, all-optical logic gates are important elements at decision points in networks. The ultrafast switches are particularly relevant to packet switched networks, although they can also be used to enhance the capabilities of wavelength-division-multiplexed channels. After reviewing recently demonstrated SDLG's and NOLM's, we provide experiments and simulations of advanced soliton logic gates that have long interaction lengths between orthogonally-polarized pulses by using low-bi fiber. The polarization-maintaining, low-bi fiber can be made by carefully wrapping fibers on aluminum mandrels without twisting: the diameter of the mandrel determines the birefringence.

We demonstrate a low-latency (i.e., in $15 \mathrm{~m}$ of fiber) soliton logic gate using lowbi fiber, and we show that the switching energy can be further reduced by using dispersion-shifted fibers. One drawback of this configuration is that the timing window is only about a pulse width wide, which means that the synchronization requirements for the input signals are quite stringent. We can remedy this difficulty by wrapping two cross-spliced lengths of low-bi fiber into a NOLM. We show that the timing window for the low-bi-NOLM can be 3.3 pulse widths wide. The lowbi-NOLM combines the best features of NOLM's and SDLG's, and its performance can be further improved by using more cross-spliced sections or using EDFA's.

This work is supported by ARPA and NSA.

\section{REFERENCES}

[1] M.N. Islam, Ultrafast Fiber Switching Devices and Systems (1992, Cambridge University Press, Cambridge, England, ISBN \# 0521431913)

[2] L.F. Mollenauer, E. Lichtman, M.J. Neubelt and G.T. Harvey, Electron. Lett. 29, 910 (1993).

[3] N.J. Doran and D. Wood, Opt. Lett. 13, 56 (1988).

[4] N.A. Whitaker, H. Avramopoulos, P.M.W. French, M.C. Gabriel, R.E. LaMarche, D.J. DiGiovanni and H.M. Presby, Opt. Lett. 16, 1838 (1991).

[5] M.N. Islam, Opt. Lett. 15, 417 (1990); M.N. Islam, C.E. Soccolich and D.A.B. Miller, Opt. Lett. 15, 909 (1990).

[6] M.N. Islam, C.-J. Chen and C.E. Soccolich, Opt. Lett. 16, 484 (1991).

[7] J.D. Moores, K. Bergman, H.A. Haus and E.P. Ippen, Opt. Lett. 16, 138 (1991).

[8] J.P. Sokoloff, I. Glesk and P.R. Prucnal, IEEE Photon. Tech. Lett. 6, 98 (1994).

[9] A.D. Ellis and D.M. Spirit, "The use of GaInAsP amplifiers for $40 \mathrm{~Gb} / \mathrm{s}$ signal processing," postdeadline paper PD-2, NGWP'93 (September 20-22, 1993, Cambridge, England). 